\title{
Predicting the Impact of Simple and Compound Life Change Events
}

\author{
Howard Wainer \\ The Bureau of Social Science Research, Inc. \\ Dianne Timbers Fairbank and Richard L. Hough \\ The University of Texas at EI Paso
}

A scale of impact for 95 life change events was determined from a sample of 4 ethnic groups. Fiftyone of these events fit a Rasch model and had the same impact in all four groups (sample free item calibration). The differences among the groups were characterized by individual parameters called "stabilities." A second study was performed that estimated a linear transformation of the impacts to yield an impact scale with ratio properties.

As people move through life, a variety of events impact upon them. What the effect of a particular life event will be depends upon two things: (1) the impact of the event, from severe (Death of Spouse) to minor (Resignation from Club or Civic Group) and (2) the stability of the person, from stoic to hysterical. A measurement model useful in characterizing life events must be able to measure both aspects.

Events frequently occur in combination; therefore, to describe the impact of a compound event properly, the measurement model must have

1. Interval properties

2. A proper origin

3. A known concatenation rule.

APPLIED PSYCHOLOGICAL MEASUREMENT

Vol. 2, No. 3 Summer 1978 pp. 311-320

(c) Copyright 1978 West Publishing Co.
There have been some attempts to describe the impact of life events (e.g., Holmes \& Rahe, 1967) as well as to ascertain the impact of compound events. The measurement models implicitly used neither allow for individual differences (stabilities) nor have the characteristics listed above. It would seem that the set of individual difference models derived from classical scaling could be useful in solving this problem.

The present research has as its goals the scaling of life events and the study of the relationship between those life events and illness. Concern thus far has been with the scaling of the events. The analysis of data from two samples-a community sample randomly drawn and a convenience sample of students-will be reported here with emphasis upon the student data. The community sample was composed of 355 residents between the ages of 20 and 60 living in twin cities of El Paso, Texas, and Ciudad Juarez, Chihuahua, Mexico. The El Paso dwellings were selected randomly from the city directory; in Juarez, a multistage area sample was drawn. The adults interviewed were selected using procedures suggested by Kish (1965).

\section{Data Collection}

The first stage of the study involved asking the 355 community residents to rate 95 life events on a 7 -point scale, the categories of which ranged 
from "events requiring least change" to "events requiring most change." The sample of 355 respondents was further subdivided into four demographic groups: (1) White-Americans living in El Paso, (2) Mexican-Americans living in El Paso, (3) Mexicans living in El Paso, and (4) Mexicans living in Juarez. An ordered category latent trait analysis (Rasch, 1960; Anderson, 1972) of all four groups both separately and aggregated yielded a reduced set of 51 items which had the following properties:

1. They fitted the model in the aggregate data.

2. They fitted the model in all of the subgroups.

3. Their scale values were not significantly different in any of the sub-samples.

Thus, for these 51 items there was a scale with all the interval properties associated with the Rasch measurement model, including samplefree item calibration. Analyses of pilot samples clearly indicated that using all seven categories in the analysis was clearly too many-the model simply did not fit. The categories were then collapsed from seven to five and finally to three. Using three ordered categories (in which the analyzed Category 1 was composed of the original Categories 1 and 2; Category II, the original Categories 3, 4, and 5; and Category III, the original Categories 6 and 7) yielded a good fit to the model. The analyses reported here used these three collapsed categories. (For a clear description of the use of the Rasch model for ordered categories, see Andrich, 1975, 1976.)

Sample-free item calibration is of great importance in measuring differences across ethnic groups. This application of latent trait analysis requires a slight change in usual vocabulary. The item scale values, which in mental testing jargon are called "difficulties" are here called impacts. The person scores which are usually called "abilities" are here called stabilities. Thus, the effect of an item is a function of the difference between its impact and the stability of the person experiencing it. Following the Rasch model, the function is logistic.
The 51 events and their associated impacts are shown in Table 1. The standard errors associated with these impacts ranged from a high of .33 for events in the tails of the distribution to .16 for those in the middle, with a median of .21 .

The second stage of the study involved choosing 5 life events from among the 51 that were scaled in the community data and which were reasonably representative of the entire range of impacts. These are shown in Table 2, with their impacts as determined from the latent trait analysis.

A paired comparison questionnaire was formed in which various combinations of these five events were presented in contrast to other combinations. ${ }^{1}$ For example, the combination event $A B$ was compared with the individual event $C$ and also the combination BE. A balanced incomplete block design was used (Cochran \& Cox, 1957) to make the number of paired comparisons reasonable and to reduce the number of trials in which the choice was completely obvious (i.e., Spouse Unfaithful-Start Own Business compared to Start Own Business). Thirty-three White-American students and 41 Mexican-American students were requested to choose which event(s) had the greatest impact. Order of presentation was randomized.

\section{Data Analysis}

\section{White-American Data}

Following the analysis model for paired comparisons data originally developed by Thurstone (1927, Case V) and specified for this situation by Bock and Jones (1968, p. 260), the scale values for each of the events and for nine compound events were estimated jointly. These estimates are shown in the first column of Table 3, labelled $S V$. The origin and unit of measure-

'The combination Change in Responsibilities at Work -Start Own Business was not asked because this combination seemed to change the character of Change in Responsibilities at Work. 


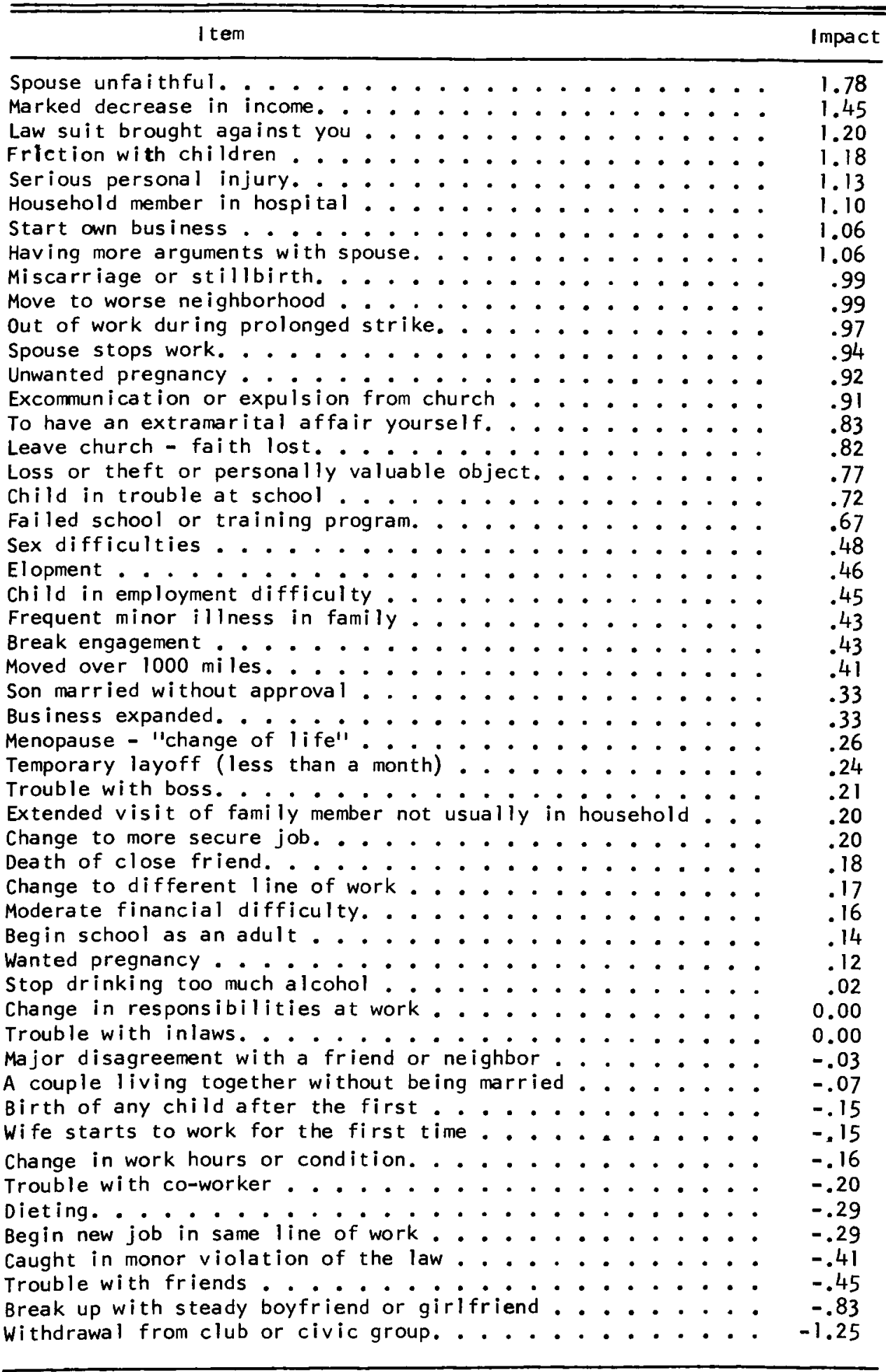


Table 2

Impact Scores of Events Used in Paired Comparisons

Impact

A. Change in responsibilities at work

B. Frequent minor illnesses in the family

.00

C. Move to worse neighborhood

D. Start own business

E. Spouse unfaithful

ment are arbitrary, so the value of zero was assigned to event $A$ and the scale values for the other events were adjusted with reference to $A$. Thus, the least serious event, Change in Responsibilities at Work, had a scale value of zero; and the most serious event was the compound event DE (Start Own Business-Spouse Unfaithful).

The unit of measurement is arbitrary, but it is consistent across all stimuli. This is almost always appropriate for any sort of measurement; lengths can be equally well measured in meters, inches, or furlongs. It remains to find a suitable origin within this metric which yields maximum agreement of compound $S V$ from simple. To do this, the testable assumption shall be made that the proper concatenation rule for these scale values is addition. If this is true, and $K$ is the proper origin, then the scale value $S V_{i}$ for event $i$ is equal to

$\mathrm{sv}_{\mathrm{i}}+\mathrm{K}$.

Similarly, the scale value for event $j$ is $s v_{j}+K$, and it follows that the scale value for compound event $i j$ is $s v_{i j}+K$. Therefore, $K$ can be estimated (if the concatenation rule is addition) by noting that

$s v_{i j}+K=\left(s v_{i}+K\right)+\left(s v_{j}+K\right)$.

According to this equation, the least squares estimate of $K$ can then easily be found from the data shown in Table 3. This estimate was 1.24. The values obtained for the new scale with an empirical origin are denoted $s_{i}{ }^{*}$ and are therefore equal to $S V_{i}+1.24$.

The success of utilizing an additive model for the combination of events can be evaluated by comparing the $s_{i j}$ 's with their estimates from the separately estimated $s_{i}{ }^{*}$ and $s_{j}{ }^{*}$. These predicted compound scale values are shown in the next column of Table 3 , labelled $s_{i}{ }^{*}+s_{j}{ }^{*}$. The correlation between the actual compound scale values and their predicted values from the single events was .95. More importantly, the median absolute deviation (MAD) of predicted compound scale value from observed scale value was .24 logits. This close fit supports the notion that a measurement model that is additive in a logit scale is reasonable, but does not confirm it.

Events in both analyses are in an additive metric though with, perhaps, different origins and units, which are arbitrary. An examination of those items common to both scales can yield an indication that they appear to be different only in unit and origin if the subjects in the respective analysis saw the items as having the same relative impacts. Since the paired comparison data have been rescaled to give a suitable origin, the latent trait scale must be rescaled to bring it, if possible, into conformation.

This procedure can be utilized to transform all of the impacts obtained from the latent trait analysis into a metric having ratio scale properties. To do this, the impacts for the five events shown in Table 2 were regressed on their scale values corrected for origin $\left(s^{*}\right)$. The best linear transformation for this is

$$
I_{i}^{*}=1.06+.96\left(I_{i}\right)
$$

where $I_{i}$ is the impact of event $i$ obtained from the latent trait analysis and $I_{i}^{*}$ is the impact of event $i$ corrected for origin. 
Table 3

Estimates of Scale Values for White-Americans Obtained From Paired Comparison Data

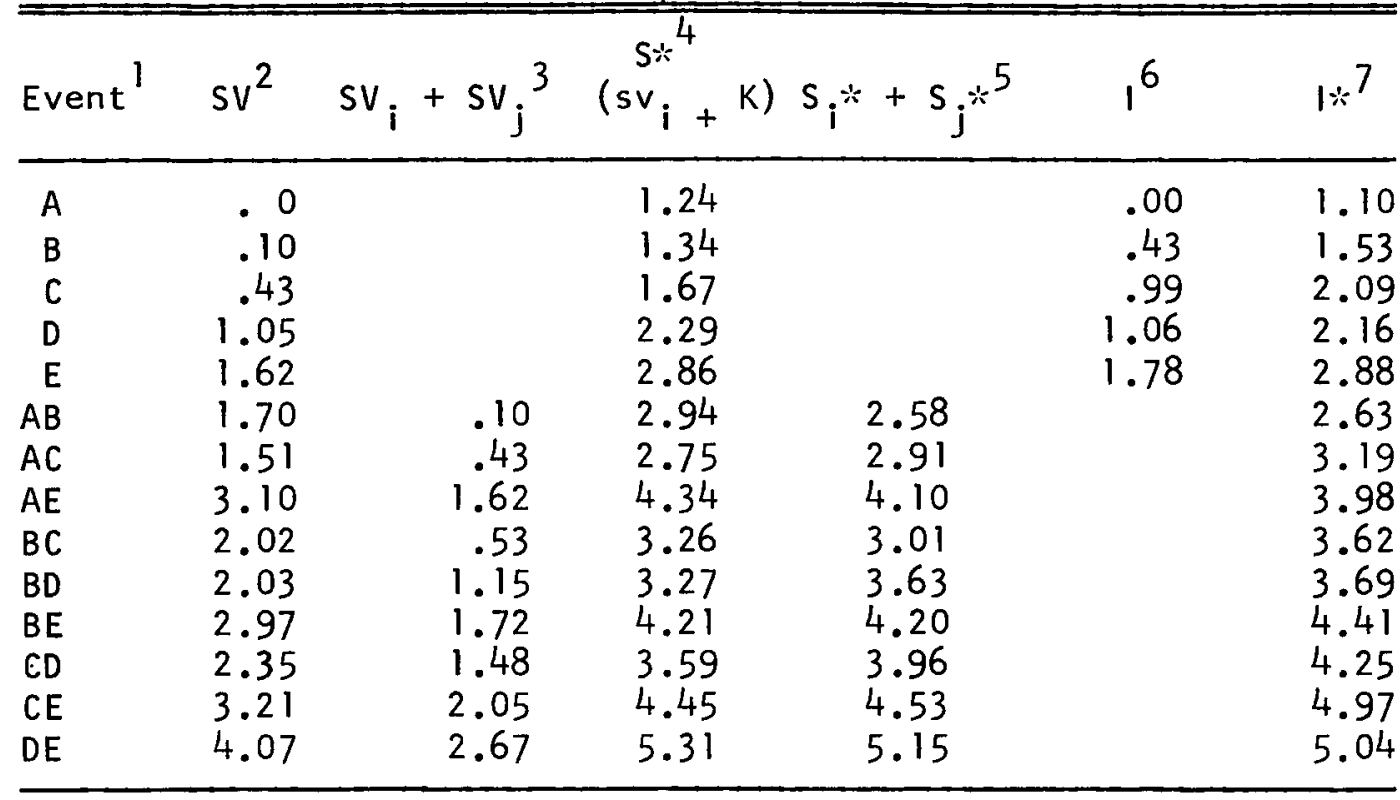

'A-"Changes in responsibilities at work'; B-Frequent minor illnesses in the family"; C-"Move to worse neighborhood"'; $D$ "Start own business"; E-"Spouse unfaithful"; $A B$ through DEcompound events.

${ }^{2}$ SV-Life Event Impact Scale Value Estimates from Paired Comparisons.

${ }^{3} S V_{i}+S V_{j}$-Predicted compound scale values from the individual Item Scale Value Estimates.

${ }^{4} \mathrm{~S} *\left(S V_{i}+K\right)-S c a l e$ Values Assuming the Concantenation Rule is addition.

${ }^{5} \mathrm{~s}_{j} *$-Predicted compound scale values from the individual items Scale Values Assuming the Concantenation Rule is addition.

61-Impact Estimate of Events Obtained from the Latent Trait Analysis.

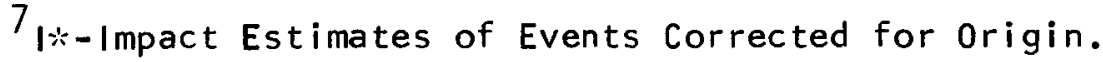


The sample size was certainly modest in this experiment; hence, reporting Equation 3 with two decimal places is not truly justified. Rounding this result to one decimal is first, more justifiable and second, more robust (Wainer \& Thissen, 1976). This yields Equation 4

$I_{1}^{*=1.1+1.0\left(I_{i}\right)}$

where $I^{*}$ and $I$ have the same meaning as before. Shown in the last column of Table 3 are the values for $I^{*}$ for the compound life events as well as predicted impacts for the nine compound events. The correlation between the predicted compound events' impact from the latent trait analysis and their actual scale values obtained from experimental measurement was .94 , with MAD equal to .36 logits. This gives hope that the measurement scheme as well as an additive model in this metric seem reasonable.

\section{Mexican-American Data}

The same analyses were repeated on data gathered from 41 Mexican-American students. The results of these analyses are shown in Table 4. The scale values obtained for the five events and nine compound events are shown in the column marked ' $S V$.' Estimated scale values for the compound events obtained by adding their component's scale values are shown in the column marked ' $s v_{i}+s v_{j \cdot}$ ' A constant was estimated which minimized the squared residual between these two columns. This constant was .45 . The scale values obtained by shifting the origin of this scale through the addition of .45 are shown in the column marked ' $S$ *'

The efficacy of this additive constant is seen when one examines the relationship between these experimentally determined scale values for compound events and their predicted value, obtained by adding their components (corrected for origin). These predicted values are shown in the column marked ' $S_{i}+S_{j}{ }^{*}$.' The correlation between the actual compound scale values and their values predicted from the single events was .93, with MAD equal to .12. The impacts obtained from the latent trait analysis (the univer- sal impacts) were regressed against the $S^{*}$ values, resulting in the regression equation.

$$
I_{i} *=.3+.6\left(I_{i}\right) \text {. }
$$

These linearly transformed impacts are shown under the column marked ' $I^{*}$,' next to the simple events. The values of the compound events are predicted from the simple events by adding their $I^{*}$ values. The MAD of the predicted compound impacts from observed scale value was .15 .

\section{Discussion}

An attempt to establish the measurement of the impact of life change events on a ratio scale was made. The extent to which this was successful is seen in the MADs. The measurement scale for the White-Americans spanned more than four logits, and the MAD was .36. This is about a $9 \%$ error, which is somewhat large. Nevertheless, the differences in experimental technique, analysis scheme, and sampling error tend to moderate this difference.

The Mexican-American data fit about the same, with a MAD of .15 logits over a 1.82 logit scale, or about $8 \%$. These results are encouraging. The accuracy with which the impacts derived from the latent trait analysis predict the empirical scale values of the compound events can be seen in plots shown in Figures 1 and 2. In both samples the choice of an additive logistic model seems to be plausible, although there appear to be significant departures from linearity in the Mexican-American sample. These departures are small, but the trends in the residuals are significant.

Although the conclusions must be qualified somewhat, the results of this pilot study are promising. One of the purposes of the study was to see if the impacts obtained from a Rasch type latent trait analysis related well to scale values obtained through a different procedure. The measurement model underlying both data collection schemes was very similar in that the Rasch model posits a logistic response function with equal slopes, as does the Bradley-Terry- 
Table 4

Estimates of Scale Values for Mexican-Americans obtained From Paired Comparison Data

\begin{tabular}{|c|c|c|c|c|c|c|}
\hline Event ${ }^{1}$ & $s v^{2}$ & $s v_{i}+s v_{j}^{3}$ & $\begin{array}{c}S *^{4} \\
(s v i+k)\end{array}$ & $s_{i}+s_{j} *^{5}$ & $1^{6}$ & $1 \div 7$ \\
\hline$A$ & . 0 & & .45 & & .00 & .30 \\
\hline B & .13 & & .58 & & .43 & .56 \\
\hline C & .20 & & .65 & & .99 & .89 \\
\hline D & .53 & & .98 & & 1.06 & .94 \\
\hline$E$ & 1.16 & & 1.61 & & 1.78 & 1.37 \\
\hline$A B$ & .37 & .13 & .82 & 1.03 & & .86 \\
\hline$A C$ & .84 & .20 & 1.29 & 1.10 & & 1.19 \\
\hline$A E$ & 1.55 & 1.16 & 2.00 & 2.06 & & 1.67 \\
\hline$B C$ & 1.15 & .33 & 1.60 & 1.23 & & 1.45 \\
\hline BD & 1.23 & .66 & 1.68 & 1.56 & & 1.50 \\
\hline $\mathrm{BE}$ & 1.69 & 1.29 & 2.14 & 2.19 & & 1.93 \\
\hline$C D$ & 1.14 & .73 & 1.59 & 1.63 & & 1.83 \\
\hline$C E$ & 1.85 & 1.36 & 2.30 & 2.26 & & 2.26 \\
\hline$D E$ & 1.83 & 1.69 & 2.27 & 2.59 & & 2.31 \\
\hline
\end{tabular}

'A-"Changes in responsibilities at work"; B-"Frequent minor illnesses in the family"; C-l'Move to worse neighborhood"'; D-"Start own business"; E-"'Spouse unfaithful"; $A B$ through De-Compound events. ${ }^{2}$ SV-Life Event Impact Scale Value Estimates from Paired Comparisons. ${ }^{3} s v_{j}+s v_{i}$-Predicted compound scale values from the individual Item Scale Values Assuming the Concantenation Rule in addition. ${ }^{4} \mathrm{~S} *\left(\mathrm{SV}_{\mathrm{j}}+\mathrm{K}\right)$-Scale Values Assuming the Concantenation Rule in addition. ${ }^{5} S_{i} * S_{j} *-P$ redicted Compound Scale Values from the Individual Items 6-Impact Estimate Events Obtained from the Latent Trait Analysis. ${ }_{1} *$-Impact Estimates of Event Corrected for Origin.

Luce model used for the scale values. The conclusion was that even though these two schemes are not identical, they are very similar. Another purpose of this study was to determine whether or not the current practice of assessing the impact of a compound event by adding up the impacts of its components is justified; the data suggest that it is.
Thus, it can be said that the determination of the impact of compound events can be determined by obtaining impacts through latent trait analysis and then transforming them with Equations 4 or 5 . Note that the transformation appropriate for the White-Americans is different from the one required for the Mexican-Americans. This is a way of characterizing each group. 


\section{Figure 1}

Impact of Some Life Events for 33 White-Americans

Along the $X$-axis are the linearly transformed values of the impacts of five life events and the predicted values of the impact of some compound events. The $Y$-axis has the empirically determined scale values for these events.

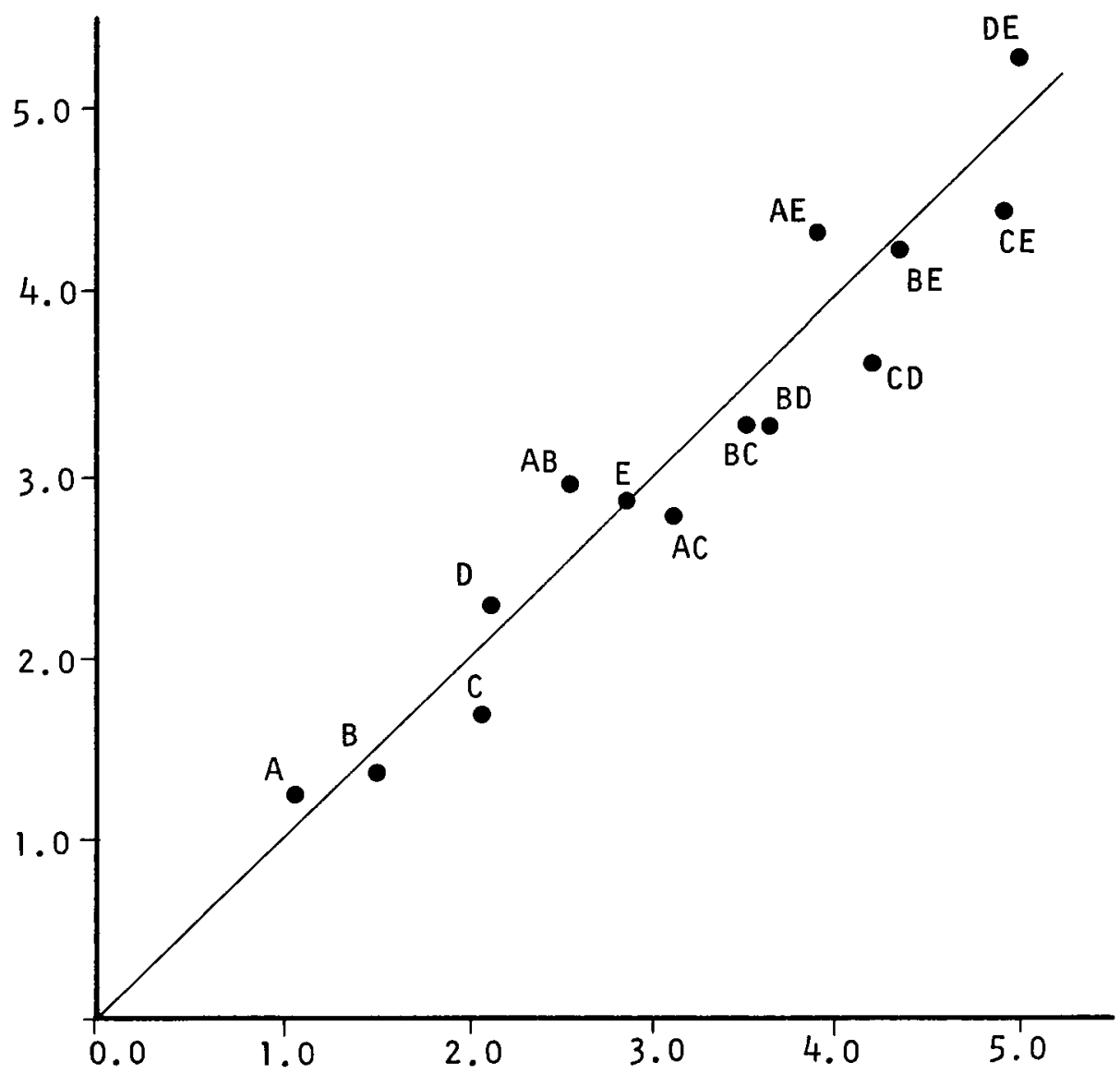

Moreover, this means that an experimental investigation to determine the zero point and concatenation rule should be carried out on a subset of the items before the impacts obtained from the interval scale values yielded by the latent trait analysis of all items can be used.

The inaccuracies of this scheme are divided between the incorrectness of the model and the small samples in the paired comparison experiment. These inaccuracies are clearly shown in the figures and are also reflected in the estimate of the lowest scale value obtainable. Substitute a value of zero for $I^{*}$ in Equation 4. Solving Equation 4 will then indicate the lowest impact obtained: the impact associated with an event that causes zero change. When this is done for White-Americans, an event with an impact of -1.1 is seen as absolute zero, or no change. This impact is lower than all events in the universal index except Withdrawal from Club or Civic Group, which has an impact of -1.25 . It also has a relatively larger error of fit. The difference be- 
Figure 2

Impact of Some Life Events for 41 Mexican-Americans

Along the $X$-axis are the linearly transformed values of the impacts of five life events and the predicted values of the impact of some compound events. The $Y$-axis has the empirically determined scale values for these events.

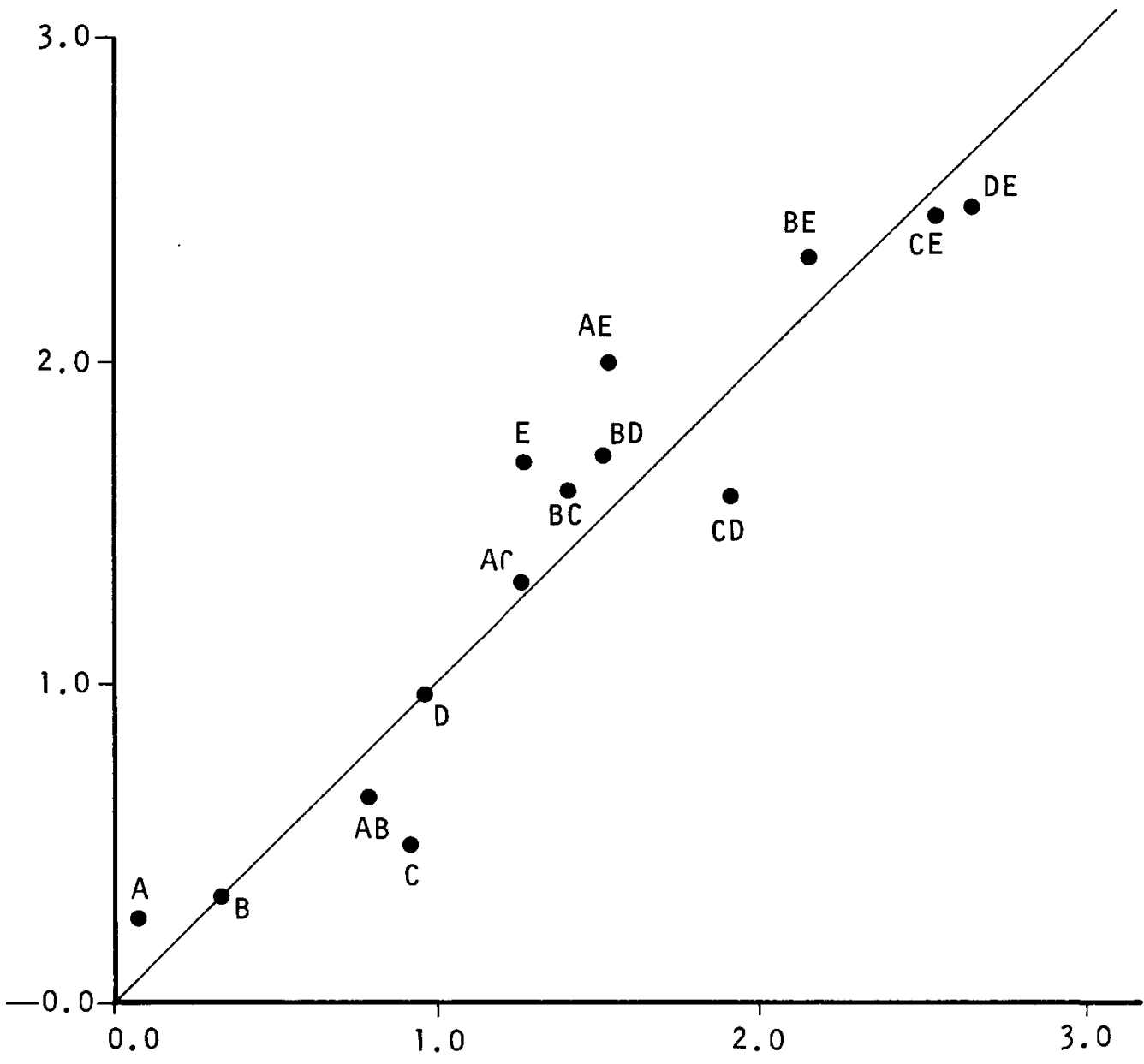

tween -1.25 and -1.1 is an indication of the model's accuracy. The corresponding zero point for Mexican-Americans is -.5 , which is considerably farther from -1.25 . Perhaps with larger samples and more thorough experimentation, these errors can be reduced and this measure can be improved.

The advisability of using student samples to supplement a community sample may be questioned, since there are obvious status and age differences between the two. This procedure was justified by the fact that the scale shown in Table 1 contained only items that scaled similarly across all the members of the community sample, including low status Mexicans and high status White-Americans, the young and the old, and so forth. Therefore, the scale is fundamentally a universal impact scale for Mexican, Mexican-American, and White-American residents of El Paso-Juarez. Further, to the extent 
that students are not comparable to the community sample, the results from the two sets of data-community and student-would have diverged instead of tending to converge as, in fact, they did.

There are implications of this study for the scoring of life events in attempting to assess the impact of life changes upon illness. The usual procedure in computing a life change score is to add means obtained from categorical estimations. This procedure is probably wrong, if the data presented in this paper are correct, because the additive model works for the latent trait values, which are exponents. This means that raw data should be multiplied, not added as in the traditional procedure; there are nonlinearities in the tails of our distributions, which are more emphatic still with raw scores.

Currently, a larger set of student data is being examined to determine if the judgments presented in this paper still hold. If these judgments are supported, this suggests that life changes can be measured more accurately than is current practice. It is hoped that with the improvement of measurement, the life change variable may be more predictive of physical and psychological disorder onset than has been found in previous research.

These procedures also bear on a larger topic of discussion in social science. The proper response, when faced with low predictive capacity of presumed independent variables, is not only to search for new independent variables and/or alternative theoretical models; the proper recourse may also be to use more sophisticated measurement models.

\section{References}

Anderson, E. B. The numerical solution of a set of conditional likelihood equations. Journal of the Royal Statistical Society, B, 1972, 34, 42-54.

Andrich, D. The Rasch multiplicative binomial model: Applications to attitude data (Research Report No. 1). University of Western Australia, Department of Education, Measurement and Statistics Laboratory, 1975.

Andrich, D. Scaling attitude items constructed and scored in the Likert tradition: $A$ unification with Thurstone scaling. Unpublished paper, 1976.

Bock, R. D., \& Jones, L. V. The measurement and prediction of judgment and choice. San Francisco: Holden Day, 1968.

Cochran, W. G., \& Cox, G. M. Experimental designs. New York: Wiley, 1957.

Holmes, T. H., \& Rahe, R. H. The social adjustment rating scale. Journal of Psychosomatic Research, 1967, 11, 213-218.

Kish, L. Survey sampling. New York: John Wiley \& Sons, 1965.

Thurstone, L. L. A law of comparative judgment. Psychological Review, 1927, 34, 278-286.

Wainer, H., \& Thissen, D. Three steps toward robust regression. Psychometrika, 1976, 41, 9-34.

\section{Acknowledgements}

The authors gratefully acknowledge support for the research from which this paper stems from the Center for Epidemiologic Research of The National Institute of Mental Health (Grant \#2 RO1 MH 26108). The Hogg Foundation for Mental Health, and the University of Texas at El Paso.

\section{Author's Address}

Howard Wainer, The Bureau of Social Science Research, Inc., 1990 M Street, N.W., Washington, DC 20036 